\title{
Momentum dependence of effective Coulomb interaction in the magnetism of nickel
}

\author{
B Sriram Shastry \\ School of Physics. University of Hyderabad, Hyderabad-500001, India
}

Received 6 November 1978, in final form 22 February 1979

\begin{abstract}
R D Lowde and C G Windsor have pointed out that good agreement between neutron scattering experiments on $\mathrm{Ni}$ and theory is possible if the effective Coulomb interaction is a mildly decreasing function of the momentum. We present a variational calculation of the wave vector-dependent susceptibility in the $t$ matrix scheme for the short-ranged one-band model. The scheme satisfies the Ward-Takahashi identities for self-energy and the vertex part. Numerical results are presented assuming an idealised bandstructure for Ni. Our computation yields results of the type suggested by experiment. We point out the reason why other theories give results which contradict experiment.
\end{abstract}

\section{Introduction}

There has been considerable interest recently in the wave vector-dependence of the magnetic susceptibility of the short-ranged one-band model (the Gutzwiller-HubbardKanamori model). Lowde and Windsor (1970) analysed neutron scattering data from metallic $\mathrm{Ni}$ in terms of a mean field-like expression for $\chi$ of this model:

$$
\chi(\boldsymbol{q}, \omega)=\chi_{0}(\boldsymbol{q}, \omega) /\left(1-U_{\text {eff }}(q) \chi_{0}(\boldsymbol{q}, \omega) / N_{\mathrm{a}}\right)
$$

where $\psi_{0}$ is the noninteracting value, $N_{\mathrm{a}}$ the number of atoms and $U_{\text {eff }}(q)$ a phenomenological effective Coulomb interaction. They found very good agreement between theory and experiment in the paramagnetic phase $\left(T>T_{\mathrm{c}}\right)$, provided $U_{\text {eff }}(q)$ was a slowly decreasing function of $q$. Osborne (1970) attempted to explain this behaviour by generalising Kanamori's $t$ matrix theory (1963) to finite wave vectors. Subsequently, inconsistencies in Osborne's treatment were pointed out (Shastry 1976). It was shown that the $t$ matrix theory equations are amenable to a variational solution and indeed yield results which are in qualitative agreement with experiment. Very recently Haga et al (1978) have again noticed the inconsistencies of Osborne's treatment but conclude that the corrected results are in disagreement with experiment: they find that $U_{\text {eff }}(q)$ increases with $q$.

In this paper we present the details of our variational calculation and compare the results with those of the other authors. In $\$ 2$ we outline the formalism of the $t$ matrix theory and set up a basic set of equations for the vertex and self-energy which are guaranteed to satisfy Ward-Takahashi identities (Hertz and Edwards 1972). In $\$ 3$ we outline the variational method used to solve these equations. In $\$ 4$ we present the results of our numerical computation for the Stoner factor as well as the wave vector dependence of $U_{\text {eff }}$. In $\$ 5$ we compare our results with those of other authors and critically examine the approximations made. 


\section{The $t$ matrix theory for the susceptibility}

The susceptibility is given by

$$
\not(q)=-\mathrm{i} \operatorname{Tr}_{p_{1} p_{2}} G_{\downarrow \uparrow ; \uparrow \uparrow}^{\mathrm{II}}\left(p_{1}+q / 2, p_{2}-q / 2 ; q\right)
$$

where

$$
\operatorname{Tr}_{p} \equiv \Omega \mathrm{i} \int_{-x}^{+\infty} \frac{\mathrm{d} p_{0}}{2 \pi} \int \frac{\mathrm{d}^{3} p}{(2 \pi)^{3}} .
$$

$\Omega$ is the crystal volume, $p \equiv\left(p_{0}, \boldsymbol{p}\right), q \equiv(\omega, \boldsymbol{q})$ and

$$
\begin{aligned}
G_{\alpha \beta ; \gamma \delta}^{\mathrm{II}}\left(k_{1}, k_{2} ; q\right) & =\iiint\left\langle 0\left|T\left\{a_{\boldsymbol{k}_{1} \mathbf{x}}\left(t_{1}\right) a_{\boldsymbol{k}_{2} \beta}\left(t_{2}\right) a_{\boldsymbol{k}_{1}-\boldsymbol{q}_{\delta}}^{+}\left(t_{4}\right) a_{\boldsymbol{k}_{2}+\boldsymbol{q}_{\gamma}}^{+}\left(t_{3}\right)\right\}\right| 0\right\rangle \\
& \times \operatorname{expi}\left[k_{1}^{0}\left(t_{1}-t_{4}\right)+k_{2}^{0}\left(t_{2}-t_{3}\right)-\omega\left(t_{3}-t_{4}\right)\right] \\
& \times \mathrm{d}\left(t_{1}-t_{4}\right) \mathrm{d}\left(t_{2}-t_{3}\right) \mathrm{d}\left(t_{3}-t_{4}\right)
\end{aligned}
$$

(our notation closely follows that of Abrikosov et al 1963). We have omitted a factor of $2 \mu_{\mathrm{B}}^{2}$ from the definition of $\chi$ for convenience. We define a vertex part $\Lambda_{\mu} \equiv\left(\Lambda_{0}, \Lambda_{i}\right)$ through the relation

$G_{\downarrow}(k+q / 2) \Lambda_{\mu}(k, q) G_{\uparrow}(k-q / 2)=-\mathrm{i} \operatorname{Tr}_{p} G_{\downarrow}^{\mathrm{Il}} \uparrow_{\uparrow \downarrow \uparrow}(k+q / 2, p-q / 2 ; q) \lambda_{\mu}(p)$

where $\lambda_{\mu}(p) \equiv\left(\lambda_{0}, \lambda_{i}\right)$

$$
\lambda_{0}(p)=1 . \quad \lambda_{i}(p)=p_{i} / m
$$

and $G_{\uparrow}\left(G_{\downarrow}\right)$ is the usual spin-up (down) single-particle propagator.

The susceptibility is given in terms of the vertex part as (see figure 1(a)).

$$
\chi(q)=\operatorname{Tr}_{k} G_{\ddagger}(k+q / 2) \Lambda_{0}(k, q) G_{\uparrow}(k-q / 2) .
$$

We may set up a Bethe-Salpeter equation for the vertex part by following standard methods (e.g. see Nozieres 1964). In the $t$ matrix theory the irreducible interaction is chosen as the particle-particle ladder (see figure 1(b)). The $t$ matrix in the case of the short-ranged one-band model is (a geometric series in this case)

$$
I(p)=\left(U / N_{\mathrm{a}}\right)\left[\left[1-\left(U / N_{\mathrm{a}}\right) \operatorname{Tr}_{k} G(k) G(p-k)\right] .\right.
$$

Here $U$ is the bare Coulomb coupling constant (e.g. see Herring 1966). These diagrams dominate perturbation theory in the low-density limit, as is well known from the theory of nuclear matter. For metals such as Ni with few fermions per band ( 0.2 holes per $\mathrm{d}$ band) the importance of this approach was recognised by Kanamori (1963). The Dyson equation for self-energy in the $t$ matrix theory is (Galitskii 1958)

$$
G^{-1}(k)=G_{0}^{-1}(k)+\operatorname{Tr}_{p} G(p) I(k+p)
$$

where $G_{0}$ is the usual noninteracting Green's function $\left(k_{0}-\epsilon_{k}+\mathrm{i} \delta\right)^{-1}$ (see figure $1(c)$ ). The Bethe-Salpeter equation for $\Lambda_{\mu}$ can be written down by inspection as (figure $1(d)$ )

$$
\Lambda_{\mu}(k, q)=\lambda_{\mu}(k)+\operatorname{Tr}_{p} I(k+p) G(p+q / 2) \Lambda_{\mu}(p, q) G(p-q / 2) .
$$

(we omit the spin label on the $G$ since we will work in the paramagnetic phase). 


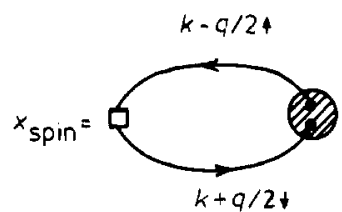

(a)
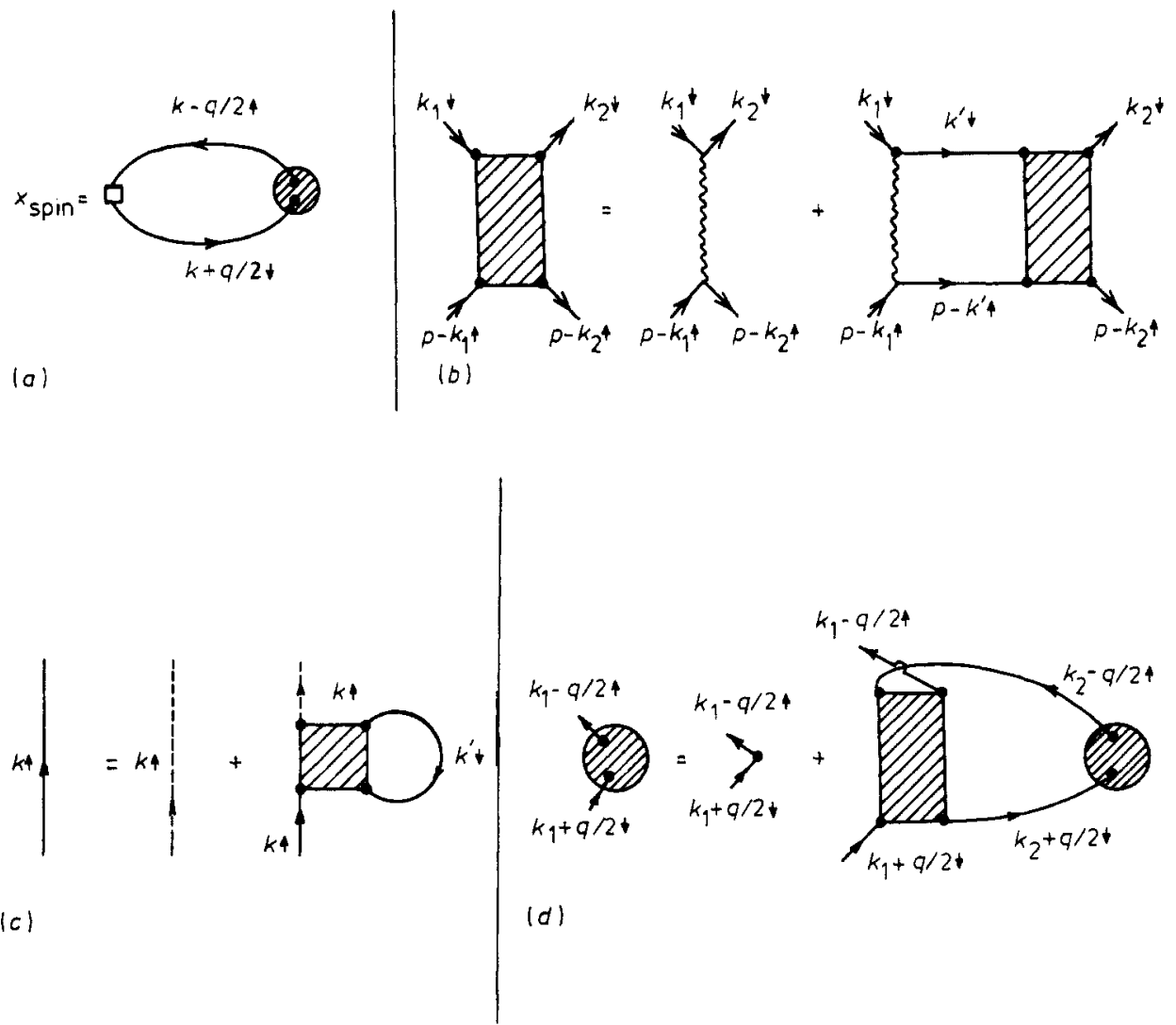

Figure 1. Feynman diagrams corresponding to the various equations necessary to calculate the susceptibility: (a), the defining equation for $\chi$ (equation (7)); (b), integral equation for the $t$ matrix (equation (8)); (c), Dyson's equation in the $t$ matrix approximation (equation (9)); (d), Bethe-Salpeter equation for the vertex part in the $t$ matrix theory (equation (10)).

We must check the consistency of equations (9) and (10) in order to satisfy spin conservation laws (Hertz and Edwards 1972). The Ward-Takahashi identity which must be fulfilled reads

$$
q^{\mu} \Lambda_{\mu} \equiv \omega \Lambda_{0}(k, q)-\sum_{i=1}^{3} q_{i} \Lambda_{i}(k, q)=G^{-1}(k+q / 2)-G^{-1}(k-q / 2) .
$$

We multiply equation (10) by $q^{\mu}$ and combine with equation (11). This yields

$$
q^{\dot{u}} \Lambda_{\mu}=q^{\mu} \hat{\lambda}_{\mu}(k)+\operatorname{Tr}_{p} I(k+p)[G(p-q / 2)-G(p+q / 2)] .
$$

Using equation (9) we see that the RHS of equation (12) is indeed

$$
G^{-1}(k+q / 2)-G^{-1}(k-q / 2) \text {. }
$$

Hence we have explicitly verified the compatibility of the self-energy and the vertex part. It should be noted that if one tries to use $G_{0}$ in place of $G$ in equations (10) and (7), the Ward-Takahashi identity is violated. 


\section{The variational principle}

The mathematical problems consists of the evaluation of $\chi(q)$ from equation (7), where the functions $G$ and $\Lambda$ are given as solutions of equations (9) and (10). A direct analytical solution seems impossible and we resort to a variational attack. Such methods were introduced by Rau and Rajagopal (1975) in another context. We set up a Lagrangian with the equations to be solved as constraints. Thus we write

$\chi_{v}=\operatorname{Tr} G_{\mathrm{t}} \Lambda_{1} G_{\mathrm{t}}+\operatorname{Tr} L_{1}\left(\Lambda_{\mathrm{t}}-1-\operatorname{Tr} I G_{\mathrm{t}} \Lambda_{\mathrm{t}} G_{\mathrm{t}}\right)+\operatorname{Tr} L_{2}\left(G_{\mathrm{t}}\left(G_{0}^{-1}+\operatorname{Tr} I G_{\mathrm{t}}\right)-1\right)$.

We have suppressed the momentum arguments to save writing. The subscripts $t$ and $v$ stand for trial and variational respectively. The Lagrange 'multipliers' $L_{1}$ and $L_{2}$ are each functions of two moments (like $\Lambda$ ) and are determined by requiring independence of $\chi_{v}$ with respect to variations of $\Lambda$ and $G$ respectively. Thus we write $G_{t}(k)=G(k)+\delta G(k)$ and $\Lambda_{t}(k)=\Lambda(k)+\delta \Lambda(k)$ and equate the coefficients of $\delta G$ and $\delta \Lambda$ to zero. This leads to equations

$$
L_{1}(k, q)=-G(k+q / 2) G(k-q / 2)+\operatorname{Tr}_{k^{\prime}} I\left(k+k^{\prime}\right) L_{1}\left(k^{\prime}, q\right)
$$

and

$$
\begin{gathered}
L_{2}(k, q)=-G(k)\left[G(k+q) \Lambda^{2}(k+q / 2, q)+G(k-q) \Lambda^{2}(k-q / 2, q)\right. \\
-G(k) \operatorname{Tr}_{k^{\prime}} I\left(k+k^{\prime}\right) G\left(k^{\prime}\right) L_{2}\left(k^{\prime}, q\right) .
\end{gathered}
$$

Comparing equations (14) and (10), we see that

$$
L_{1}(k, q)=-G(k+q / 2) \Lambda(k, q) G(k-q / 2) .
$$

Equation (15) must be solved in order to get the Lagrange function $L_{2}$. We note however that the inhomogeneous term is independent of $I$ and gives the leading contribution to $\chi_{v}$. The second term leads to terms of higher order in $I$. This term is assumed to be small in the $t$ matrix theory and hence is neglected.

We further choose $G_{t}$ to be $G_{0}$, the noninteracting Green's function, for simplicity. The advantage of working with a variational principle now manifests itself; one may confidently make approximations such as the above, since the variational principle provides suitable counter terms that minimise the error.

We still have the freedom of choosing $\Lambda_{t}(k, q)$. In this we are guided by the integral equation for $\Lambda_{0}(k, q)$ (equation (10)). Since the inhomogeneous term is independent of the first momentum, we choose $\Lambda_{t}(k, q)=\mu(q)$, i.e. a function of $q$ only, the best value being determined by minimising $\chi_{v}$ with respect to $\mu$. This gives a solution of the form of equation (1), with

$$
\%_{0}(q)=\operatorname{Tr}_{p} G_{0}(p+q / 2) G_{0}(p-q / 2)
$$

and

$$
U_{\mathrm{eff}}(q)=N_{\mathrm{a}}\left[J_{1}(q)-J_{2}(q)\right] / \chi_{0}^{2}(q)
$$

where

$J_{1}(q)=\operatorname{Tr}_{k, p} G_{0}(k+q / 2) G_{0}(k-q / 2) I(k+p) G_{0}(p+q / 2) G_{0}(p-q / 2)$

and

$$
\begin{aligned}
& J_{2}(q)=\operatorname{Tr}_{k, p} G_{0}(k+q / 2) G_{0}(k-q / 2) I(k+p) \\
& \times\left[G_{0}(p+q / 2) G_{0}(k-q / 2)+G_{0}(p-q / 2) G_{0}(k+q / 2)\right] .
\end{aligned}
$$


The terms $J_{1}$ and $J_{2}$ arise from the vertex and self-energy respectively. It is clear that the self-energy constraint gives a term of the same order as the vertex part and hence cannot be ignored. We remark that equation (18) is essentially exact for $q \rightarrow 0, \omega \rightarrow 0, \omega / q \rightarrow 0$ since in this limit the integral equation (equation (10)) for the vertex part becomes algebraic and can be trivially solved (for a spherical Fermi surface).

\section{Results}

\subsection{The t matrix for a model bandstructure}

The $t$ matrix (equation (8)) may be written in the form

$$
I(p)=\left(U / N_{\mathrm{a}}\right)\left(1+U n\left(\epsilon_{\mathrm{F}}\right) F(p)\right)^{-1}
$$

where $n\left(\epsilon_{F}\right)$ is the density of states per atom and

$$
F(p)=\frac{\Omega}{N_{\mathrm{a}} n\left(\epsilon_{\mathrm{F}}\right)} \int \frac{\mathrm{d}^{3} k}{(2 \pi)^{3}} \frac{\left(1-f_{k}\right)\left(1-f_{p-k}\right)-f_{k} f_{p-k}}{\epsilon_{k}+\epsilon_{p-k}-p_{0}+\operatorname{in}\left(f_{k}+f_{p-k}-1\right)}
$$

where $f_{k}$ is the usual Fermi function $\left(=\theta\left(\epsilon_{\mathrm{F}}-\epsilon_{k}\right)\right)$ and $\epsilon_{k}$ is the (bare) kinetic energy. The frequency $p$ may be set at $2 \epsilon_{\mathrm{F}}$ since one needs the average of $I$ with both particles near the Fermi surface (the $G$ in equations (19) and (20) are peaked at the Fermi surface).

We now introduce a somewhat crude representation for the bandstructure of $\mathrm{Ni}$. We assume parabolic bands with $\epsilon_{k}=\hbar^{2} k^{2} / 2 m^{*}$ and replace the Brillouin zone by a sphere of radius $k_{m}$ chosen such that the volumes coincide (i.e. a Wigner-Seitz construction). In terms of $n_{\mathrm{h}}$, the number of $\mathrm{d}$ band holes per band per atom, we have

$$
k_{m}=\left(2 / n_{\mathrm{h}}\right)^{1 / 3} k_{\mathrm{F}}, \quad n\left(\epsilon_{\mathrm{F}}\right)=3 n_{\mathrm{h}} / 4 \epsilon_{\mathrm{F}} . \quad k_{\mathrm{F}}=\left(12 \pi^{2} n_{\mathrm{h}}\right)^{13} a_{\mathrm{L}}^{-1}
$$

where $a_{\mathrm{L}}$ is the lattice constant of the FCC Ni lattice and $k_{\mathrm{F}}$ is the Fermi wave vector. With this bandstructure we can calculate the function $F$ analytically. We find for $p \leqslant 2$

$$
\begin{aligned}
& F(p)=F_{1}(p)+F_{2}(p) \\
& F_{1}(p)=k_{m}-\frac{1}{2}(1+p / 2)+\frac{1}{2} q \ln \left\{\frac{\left(k_{m}-q\right)}{k_{m}+q} \frac{1+q}{p / 2}\right\}
\end{aligned}
$$

and

$$
F_{2}(p)=\frac{1}{2}(p-2)+\frac{1}{2} q \ln \left(\frac{1+q}{p / 2}\right)
$$

where $q=\left(1-p^{2} / 4\right)^{1 / 2}$, and $F_{1}$ and $F_{2}$ are the particle-particle $(\mathrm{p}-\mathrm{p})$ and hole-hole (h-h) terms respectively and correspond to the terms $\left(1-f_{k}\right)\left(1-f_{p-k}\right)$ and $f_{k} f_{p-k}$ in equation (22). For $p>2$ the function $I$ may be approximated by its asymptotic form $c / p^{2}$, the constant $c$ being chosen to obtain continuity with the value for $p \leqslant 2$. This gives for $p>2$

$$
F_{1}(p)=4\left(k_{m}-1\right) / p^{2} . \quad F_{2}(p)=0 .
$$


Using the above bandstructure, we can write $U_{\text {eff }}$ (equation (18)) as

$$
U_{\text {eff }}(q)=\frac{-U}{8[W(q / 2)]^{2}} \iint \frac{\mathrm{d}^{3} \boldsymbol{k}}{2 \pi} \frac{\mathrm{d}^{3} \boldsymbol{p}}{2 \pi} \frac{[(\boldsymbol{k}-\boldsymbol{p}) \cdot \boldsymbol{q}]^{2}}{1+\operatorname{Un}\left(\epsilon_{\mathrm{F}}\right) F(|\boldsymbol{k}+\boldsymbol{p}|)} \frac{m_{q}(k)}{(\boldsymbol{k} \cdot \boldsymbol{q})^{2}} \frac{m_{q}(p)}{(\boldsymbol{k} \cdot \boldsymbol{p})^{2}}
$$

where

$$
W(x)=\frac{1}{2}+\frac{1-x^{2}}{4 x} \ln \left(\frac{1+x}{|1-x|}\right)
$$

and

$$
m_{q}(k)=f_{k-q / 2}-f_{k+q / 2} .
$$

\subsection{The uniform susceptibility and the Stoner factor}

The uniform susceptibility is of considerable interest since its divergence signals the onset of ferromagnetism. In the present scheme, the Stoner factor (defined as the dimensionless ratio $\chi / \chi_{0}$ ) can be expressed as

$$
S_{0}=\left[1-n\left(\epsilon_{\mathrm{F}}\right) N_{\mathrm{a}}\left\langle I(\boldsymbol{k}+\boldsymbol{p})\left\{1-\boldsymbol{q} \cdot \nabla_{\boldsymbol{k}} \epsilon_{\boldsymbol{k}} / \boldsymbol{q} \cdot \nabla_{\boldsymbol{p}} \epsilon_{\boldsymbol{p}}\right\}\right\rangle\right]^{-1} .
$$

The angular bracket represents an average over the Fermi surface

$$
\left\langle>\equiv(2 \pi)^{-6}\left[\Omega / N_{\mathrm{a}} n\left(\epsilon_{\mathrm{F}}\right)\right]^{2} \iint \frac{\mathrm{d} s_{k} \mathrm{~d} s_{p}}{\left|\nabla_{k} \epsilon_{k}\right|\left|\nabla_{p} \epsilon_{p}\right|} .\right.
$$

The second term in the braces in equation (31) arises from the self-energy correction and was neglected by Kanamori (1963) in his pioneering work. It is therefore interesting to estimate the error made by its neglect albeit in a simplified model. Hence we also compute the function

$$
S_{1}=\left[1-n\left(\epsilon_{\mathrm{F}}\right) N_{\mathrm{a}}\langle I(k+p)\rangle\right]^{-1} .
$$

Kanamori made yet another technical assumption (termed as dangerously crude by Herring 1966). This assumption amounts to setting $k$ and $p$ equal to zero and simply omitting the averaging in equation (32) (this approximation is very convenient if one is interested in a crude estimate of the effect of the density of states function on the occurrence of ferromagnetism, which was Kanamori's main objective). This leads to the function

$$
S_{2}=\left\{1-\frac{U n\left(\epsilon_{\mathrm{F}}\right)}{1+\operatorname{Un}\left(\epsilon_{\mathrm{F}}\right)\left[\left(2 / n_{\mathrm{h}}\right)^{1 / 3}-1\right]}\right\}^{-1} .
$$

Note also that a naive mean field calculation gives

$$
S_{\mathrm{MF}}=\left(1-U n\left(\epsilon_{\mathrm{F}}\right)\right)^{-1} \text {. }
$$

The functions $S_{0}$ and $S_{1}$ can be easily computed (being reducible to single-variable integrations) for various values of $U n\left(\epsilon_{\mathrm{F}}\right)$ and $n_{\mathrm{h}}$. In figure 2 we compare the inverses of various Stoner factors for $n_{\mathrm{h}}=0.2$ as a function of $U n\left(\epsilon_{\mathrm{F}}\right)$. Thus every improvement in the approximation leads to a smaller Stoner factor and hence a lesser tendency towards ferromagnetism. If we increase $n_{\mathrm{h}}$, we find that ferromagnetism sets in (at $U=x$ ) for the following values of $n_{\mathrm{h}}: S_{2}, S_{1}$ and $S_{0}$ diverge for $n_{\mathrm{h}}=0.25,0.35$ and 0.42 respectively. This result must be treated with some caution since the low-density assumption underlying the $t$ matrix approach is no longer valid for 


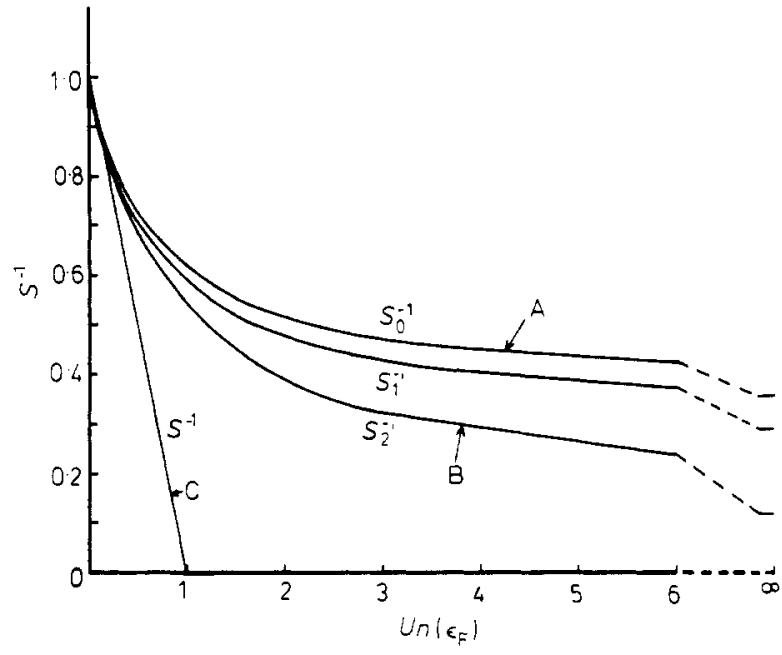

Figure 2. The inverse Stoner enhancement factor in various approximations: A, present work; B, Kanamori (1963); C. mean field approximation.

large values of $n_{\mathrm{h}}$. We may however infer that the Kanamori criterion could well overestimate the tendency towards ferromagnetism systematically.

\subsection{The wave vector dependence of $U_{\text {eff }}(q)$}

In order to perform the six-fold integrals in equation (28) we use the following useful algorithm valid for arbitrary functions $(q \leqslant 2)$ :

$$
(2 \pi)^{-1} \int \mathrm{d}^{3} k m_{\boldsymbol{q}}(k) g(\boldsymbol{k}, \boldsymbol{q})=(q / 2) \int_{-1}^{1} \mu \mathrm{d} \mu \int_{-1}^{1} k^{2}(x, \mu) \mathrm{d} x \int_{0}^{2 \pi} \frac{g(\boldsymbol{k}, \boldsymbol{q})}{(\mathrm{d} \phi / 2 \pi)} .
$$

Here we have introduced spherical polar coordinates for $k\left(k, \cos ^{-1} \mu, \phi\right)$, chosen the $z$ axis along $q$ and introduced $x$ through the relation

$$
k(x, \mu)=\frac{1}{2} q x|\mu|+\left[1+\left(q^{2} / 4\right)\left(\mu^{2}-1\right)\right]^{1 / 2} .
$$

Using this algorithm for both momenta. we find

$$
\begin{aligned}
U_{\text {eff }}(q)=-\frac{U}{32[W(q / 2)]^{2}} \iiint_{-1}^{1} \int\left(\mathrm{d} \mu_{1} / \mu_{1}\right)\left(\mathrm{d} \mu_{2} / \mu_{2}\right) \mathrm{d} x_{1} \mathrm{~d} x_{2} \\
\quad \times\left[k\left(x_{1} \mu_{1}\right)-k\left(x_{2} \mu_{2}\right)\right]^{2} \int_{0}^{2 \pi} \int_{0}\left(\mathrm{~d} \phi_{1} / 2 \pi\right)\left(\mathrm{d} \phi_{2} / 2 \pi\right)\left[1+U n\left(\epsilon_{\mathrm{F}}\right) F\left(\boldsymbol{k}_{1}+k_{2}\right)\right]^{-1} .
\end{aligned}
$$

The integrals were computed by using the Gauss-Legendre and Gauss-Chebyshev quadratures (e.g. see Abramowitz and Stegun 1965). (The apparent divergences in the integral from $\mu_{1}$ and $\mu_{2} \rightarrow 0$ are handled automatically in this method since the abscissae are symmetrical about zero.) Convergent results were obtained by using six-point quadratures for each variable.

In figure 3 we display the computed $U_{\text {eff }}(q) n\left(\epsilon_{\mathrm{F}}\right)$ as a function of $q / 2 k_{\mathrm{F}}$ for various values of $U n\left(\epsilon_{\mathrm{F}}\right)$. The number of holes/band/atom $\left(n_{\mathrm{h}}\right)$ is set at $0 \cdot 2$, and only particle-particle (p-p) scattering considered (i.e. $F_{2}$ is neglected in equation (24)). 


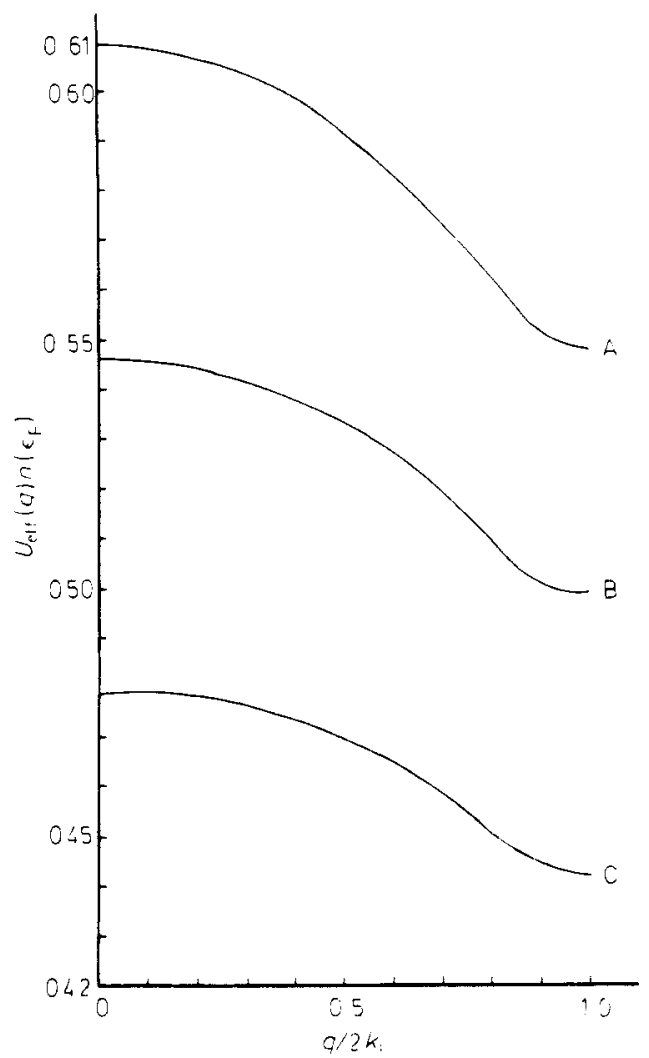

Figure 3. The effective Coulomb interaction function for various values of $\ln \left(\epsilon_{\mathrm{F}}\right)$. Only p. $p$ terms are retained and $n_{\mathrm{h}}$ set at $0 \cdot 2$. A $\ln \left(\epsilon_{\mathrm{F}}\right)=15:$ B. $\ln \left(\epsilon_{\mathrm{F}}\right)=4:$ C. $U n\left(\epsilon_{F}\right)=2$.

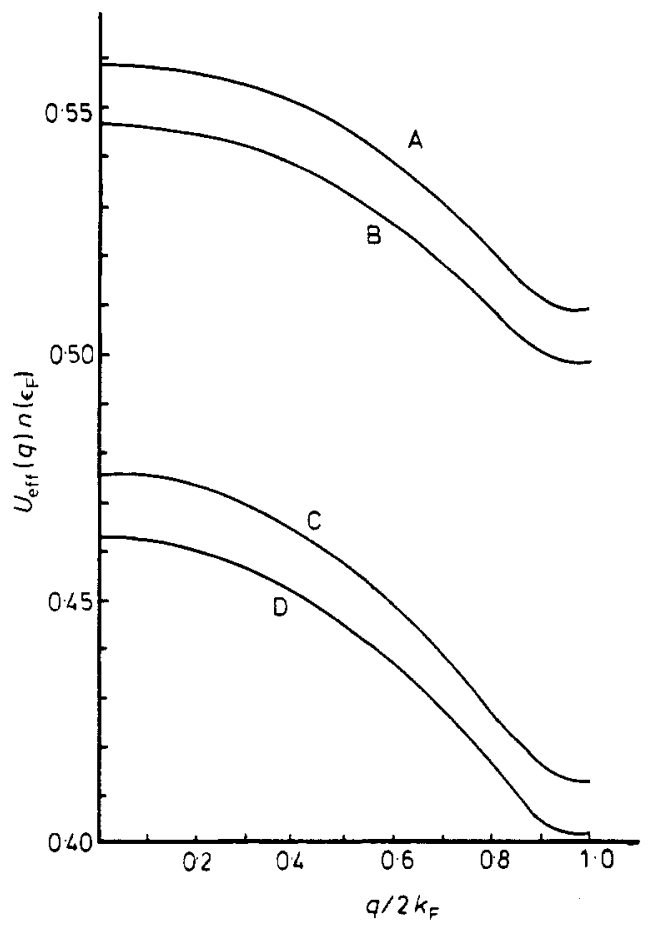

Figure 4. The effective Coulomb interaction function for different values of $n_{h}$ In all curves $U n\left(\epsilon_{\mathrm{F}}\right)=4$. Curves $\mathrm{A}$ and $\mathrm{B}$ have only $p-p$ terms; curves $C$ and $D$ have both $\mathrm{p}-\mathrm{p}$ and $\mathrm{h}-\mathrm{h}$ terms. A, $n_{\mathrm{h}}=0.21$; B, $n_{h}=0.2 ; C . n_{h}=0.2 ; D, n_{h}=0.1866$. 
The momentum dependence is seen to be very mild, becoming more pronounced for large $U n\left(\epsilon_{\mathrm{F}}\right)$.

In order to investigate the role of hole-hole $(\mathrm{h}-\mathrm{h})$ scattering and of the sensitivity to $n_{\mathrm{h}}$, in figure 4 we plot $U_{\text {eff }}(q)$ as a function of $q / 2 k_{\mathrm{F}}$ for various values of the parameters $\left(U n\left(\epsilon_{\mathrm{F}}\right)\right.$ is fixed at 4$)$. Curves marked A and B correspond to $\mathrm{p}-\mathrm{p}$ scattering and differ only in $n_{\mathrm{h}}$. It is seen that the effect of increasing $n_{\mathrm{h}}$ is to shift $U_{\text {eff }}$ upwards. Curves $C$ and $\mathrm{D}$ correspond to $\mathrm{p}-\mathrm{p}$ and $\mathrm{h}-\mathrm{h}$ terms. The inclusion of $\mathrm{h}-\mathrm{h}$ terms thus reduces the magnitude of $U_{\text {eff }}$ and also accentuates the $q$ dependence.

\section{Conclusions}

Lowde and Windsor (1970) point out that a calculation due to Osborne (1970) gives too large a $q$ dependence to $U_{\text {eff }}(q)$. Osborne's new calculation is ostensibly a generalisation of Kanamori's calculation to finite wave vectors. It must be pointed out, however, that the generalisation is incorrect and based upon the following misunderstanding. Osborne starts with essentially equation (32) which gives $U_{\text {eff }}(q=0)$. As mentioned earlier, Kanamori made an assumption of convenience, replacing $k$ and $p$ by zero. Osborne has taken $q$ to stand for $k-p$ and Taylor expands equation (32) about zero to obtain a ' $q$ dependence'. It should be clear, however, that this procedure is incorrect. Equation (32) only gives the uniform susceptibility and does not have enough information to yield $U_{\text {eff }}(q)$. One must necessarily solve equations for the vertex part for finite wave vectors to achieve the desideratum.

As mentioned in the Introduction, Haga et al (1978) have recently re-examined the $t$ matrix approach and have solved the integral equations by numerical methods. Their conclusion. however, is the opposite of ours: they find that $U_{\text {eff }}(q)$ increases with $q$ and hence state that there is a contradiction between theory and experiment. The resolution of this contradiction lies in the observation ( $T$ Kato 1978, private communication) that they have neglected the self-energy corrections. This amounts to dropping the Dyson equation constraint in our variational approach (and hence the neglect of the $J_{2}$ term in equation (18)). This procedure is clearly unjustified since spin conservation laws or the Ward-Takahashi identities (Hertz and Edwards 1972) are violated. (This is clear from \$2.) Also, our solution explicitly shows that the contributions from the self-energy corrections are of the same (numerical) order of magnitude as the vertex corrections, therefore giving rise to the possibility of cancellations.

We therefore conclude that the $t$ matrix equations for the self-energy and vertex part obeying the spin current conservation laws (or the Ward-Takahashi identities) yield a $U_{\text {eff }}(q)$ which decreases mildly with $q$ in accordance with experiments analysed by Lowde and Windsor (1970).

\section{Acknowledgments}

I thank Professors A K Rajagopal and C K Majumdar for their interest in this work. I thank Professor Kato for informing me of his work prior to publication.

\section{References}

Abramowitz A and Stegun 11965 Handhook of Mathematical Functions (New York: Dover)

Abrikosov A A. Gorkov L P and Dzyaloshinskii I E 1963 Methods of Quantum Field Theory in Statistical Phys. (Englewood Cliffs, NJ: Prentice Hall) 
Galitskii V M 1958 Sor: Phy'--JETP 34151

Haga I. Kato T and Aisaka T 1978 Prog. Theor. Phys, in press

Herring C 1966 Magnetism ed G T Rado and H Suhl (New York: Academic Press) vol 4 p 209

Hertz J A and Edwards D M 1972 Phys. Rev. Lett. 281334

Kanamori J 1963 Prog. Theor. Phys. 30275

Lowde R D and Windsor C G 1970 Adv. Phys. 19813

Nozières P 1964 Theory of Interacting Fermi Systems (New York: Benjamin)

Osborne C 1970 Phys. Lett. 29A 628

Rau A R P and Rajagopal A K 1975 Phys. Rev. B11 3604

Shastry B S 1976 PhD Thesis Tata Institute of Fundamental Research, Bombay 